\title{
Stability Analysis and Evaluation of Jointless Track Based on Ballast Surface Parameters
}

\author{
Hu Zhang1, Shougang Huang1, Yun Yang1, Ran Meng² \\ ${ }^{1}$ School of Transportation, Shijiazhuang Tiedao University, Shijiazhuang, China \\ ${ }^{2}$ Beijing Jiaotong University Integrated Transport Research Center, Beijing, China \\ Email: 2359435415@qq.com
}

How to cite this paper: Zhang, H., Huang, S.G., Yang, Y. and Meng, R. (2018) Stability Analysis and Evaluation of Jointless Track Based on Ballast Surface Parameters. Engineering, 10, 247-252.

https://doi.org/10.4236/eng.2018.105016

Received: April 2, 2018

Accepted: May 13, 2018

Published: May 16, 2018

Copyright $\odot 2018$ by authors and Scientific Research Publishing Inc. This work is licensed under the Creative Commons Attribution International License (CC BY 4.0).

http://creativecommons.org/licenses/by/4.0/

(c) (i) Open Access

\begin{abstract}
In formulas that use the probabilistic method to calculate the stability of the CWR, the value of the cross-resistance of the track beds hasn't fully taken into account the influence of the type of the parameter combination of the track bed, making the calculation results for a particular line undifferentiated. The analogy of the trajectory of the lateral displacement of the trackless bed is proposed. It is proposed that the lateral resistance of the track bed is also ambiguous within the allowable lateral displacement limit, and equivalent randomization is performed. Through the analysis of the characteristics of the appearance parameters of the track bed, a calculation formula containing multiple surface parameters for the lateral resistance of the track bed was established, and the values of various factors were reasonably analyzed. The purpose of this paper is to point out that in the stability evaluation formula, the value of the lateral parameters of the ballast bed lacks the consideration of the surface parameters. Based on the surface parameters, a model to evaluate the lateral resistance of the track bed is proposed. It can be seen as a refinement of the formula.
\end{abstract}

\section{Keywords}

Bed Surface Parameters, Lateral Resistance of Track Bed, Stability Analysis

\section{Introduction}

Many factors, including ballast resistance, track frame stiffness, initial bending and temperature forces [1], must be considered in the analysis of structural stability of CWR. Among them, the lateral resistance in the ballast resistance is the chief factor of maintaining the lateral stability of the line. In the theoretical analysis, the temperature force-transverse displacement relationship is often used to 
determine whether the track is unstable. So in view of the relatively simple considerations, and the difficulty of directly measuring the temperature of the rail, it does not have the conditions and basis for popularization in construction sites.

Actually, the evaluation of line stability should adopt a multi-factor evaluation method. But there is a wide variety of information, some of which even difficult to determine, that affects the size of these factors, bringing great inconvenience to actual maintenance. Some line information is accessible. For example, the track temperature can be measured with a track temperature meter. The curve radius, sleeper type, fastener type, etc. can all be picked up from the work department's account or maintenance information. Some information is uncertain, such as the original curvature of the bed, the temperature rise, and the randomness of the lateral resistance of the ballast bed.

Chen Xiufang [2] clarified the probability model of the original bending of the continuously welded rail track and the variables of its calculation. Of course, when the equivalent randomization method was analyzed, the limit equation for studying its stability could be constructed according to the specific formula. Zhang Xiangmin [3] used single-parameter analysis method to analyze the sensitivity of the parameters affecting the stability of the seamless track. It was found that the lateral resistance of the track, the original orbit of the track, and the temperature rise of the rail has a great influence on the stability and reliability, and the fastener stiffness has little effect. Zhu Zhengwang [4] used the calculation formula of the stability of the unequal wavelength CWR as the system mathematical model, analyzed the influence of the orbital parameters on the allowable temperature rise and the orbital deformation wavelength, and calculated their respective sensitivity coefficients. Li Qiuyi [5] analyzed the fuzzy randomness of the allowable lateral displacement of the track bed, and analyzed the probability distribution and statistical parameters of the allowable lateral displacement of the track using the equivalent random method of fuzzy variables.

From the perspective of probability [6], the established performance function of the seamless line stability by Chen Xiufangis as follows:

$$
\left\{\begin{array}{l}
Z=P-P \\
P=\frac{E I \pi^{2} \cdot \frac{f}{l^{2}}+E I \pi^{2} \cdot \frac{i_{0 e}}{l}+\frac{4}{\pi^{3}} \cdot Q l^{3}}{f+i_{0} l+\frac{4}{\pi^{3} R} \cdot l^{2}}
\end{array}\right.
$$

where $Z$ is the equation of state; $P$ is the bearing capacity; $P_{t}$ is the temperature force; $E I$ is the bending stiffness; $i_{0 e}$ is the original curvature ratio; $l$ is the wavelength; $Q$ is the track bed lateral resistance; $f$ is the rail curvature.

In the Equation (1), the above variables and their randomness are well considered. Among them, the original bending and temperature rise of the ballast bed can be statistically analyzed by probabilistic method, which is free from the limitation of evaluating line stability with single index of temperature force. However, when the value of the lateral resistance of the track bed was taken into 
account, the displacement was used as the only variable to fit a curve which could reflect the corresponding ballast bed resistance coefficient without considering the influence of the differences in the parameters of the track bed surface [7]. Therefore, when applied to the line in different track conditions, the equation cannot reflect the difference in reality. We have expanded the transverse resistance of the track bed in Equation (1), and we must consider the impact factors of the trackbed profile in Q's value process. Many factors, like the looseness and the grading of the ballast, the width and height of the ballast bed's shoulder, could affect the lateral resistance of the ballast bed. Therefore, it is difficult to quantify, the appearance of the ballast bed, such as the slackness of the ballast bed, one of the shape parameters corresponding to the transverse resistance of the ballast bed, making the lateral resistance somewhat fuzzy.

\section{Without Considering Bed Shape Parameters Causes Track Bed's Transverse Resistance's Ambiguity}

\subsection{Fuzziness of Lateral Resistance}

Track bed's transverse resistance is closely related to its lateral displacement. The lateral displacement value of the orbit is a random and fuzzy variable. Lateral displacements are allowed to be expressed in terms of fuzzy mathematical subsets [5], and the membership function is assumed to be $\mu_{A}(x)$.

$$
\mu_{A}(x)= \begin{cases}0 & 0 \leq x \leq a_{1} \\ \frac{\left(x-a_{1}\right)}{\left(a_{2}-a_{1}\right)} & a_{1}<x \leq a_{2} \\ 1 & x>a_{2}\end{cases}
$$

where, the parameters $a_{1}$ and $a_{2}$ are determined by the limits of the track's allowable lateral displacement.

Similarly, the lateral resistance of the track bed is also ambiguous within the allowable lateral displacement. Assuming that the lateral resistance of the track bed corresponding to $a_{2}$ is $Q_{2}$, and that corresponding to $a_{1}$ is $Q_{2}$, whichallows transverse resistance to be represented by a fuzzy mathematical subset $\underset{\sim}{B}$. By making an analogy the membership function of lateral bed resistance is $\mu_{\underline{\sim}}(x)$.

$$
\mu_{B}(x)= \begin{cases}0 & 0 \leq x \leq Q_{1} \\ \frac{\left(x-Q_{1}\right)}{\left(Q_{2}-Q_{1}\right)} & Q_{1}<x \leq Q_{2} \\ 1 & x>Q_{2}\end{cases}
$$

\subsection{Fuzzy Equivalent Randomization of Lateral Resistance}

The above membership function $\mu_{B}(x)$ is a monotonically increasing function, and $0<\mu_{\underline{B}}(x)<1$. The first derivative of $\mu_{\underline{B}}(x)$ is as follows: 


$$
\frac{\partial \mu_{B}(x)}{\partial x}= \begin{cases}0 & x \leq Q_{1} \\ \frac{1}{\left(Q_{2}-Q_{1}\right)} & Q_{1}<x \leq Q_{2} \\ 1 & x>Q_{2}\end{cases}
$$

From the analysis of $\mu_{B}(x)$ and $\frac{\partial \mu_{B}(x)}{\partial x}$, characteristics of a random variable probability distribution function was found in $\mu_{B}(x)$, and that of a random variable probability density function was also found in $\frac{\partial \mu_{B}(x)}{\partial x}$, which can be viewed as a probability distribution function and a probability density function on which uniformly distributing random variables in the interval $\left[Q_{1}\right.$, $Q_{2}$ ]. Therefore, in the analysis of the reliability of the seamless line structure stability, a variable $q$ can substitute the fuzzy variable $\underset{\sim}{B}$ for probabilistic analysis with a random reliability analysis method. The probability distribution function and probability density function of the equivalent random variable $q$ are as follows:

$$
\left\{\begin{array}{l}
F_{q}(x)=\mu_{A}(x) \\
f_{q}(x)=\frac{\partial \mu_{A}(x)}{\partial x}
\end{array}\right.
$$

From Equation (5), we can see that the equivalent random variable $q$ can be described by the uniform distribution in the interval $\left[Q_{1}, Q_{2}\right]$. Its mean value and standard deviation are respectively $\mu_{q}$ and $\sigma_{q}$. So it can be known from the knowledge of probability theory:

$$
\left\{\begin{array}{l}
\mu_{q}=\frac{Q_{2}+Q_{1}}{2} \\
\sigma_{q}=\frac{Q_{2}-Q_{1}}{2 \sqrt{3}}
\end{array}\right.
$$

\section{The Lateral Resistance Considering Bed Surface Parameters}

The lateral resistance of the ballast bed is a force that prevents the track structure from buckling laterally in the ballast bed and a key influence factor of the stability of the seamless line. The resistance of the ballast bed mainly consists of the frictional resistance between the sleeper and the ballast and the shoulder-to-sleeper force of the ballast bed. There are many surface parameters affecting the lateral resistance of the track bed, which can be divided into quantifiable parameters and non-quantitative parameters. The quantifiable parameters mainly include: thickness, shoulder width, shoulder height and shoulder slope of ballast bed, which are denoted by $h, b, l$, and $n$, respectively. The degree of looseness of the ballast bed, the main aspect of non-quantitative parameters, which is related to the material and grading of the ballast, and is represented by the coefficient $\xi$. The transversal resistance equivalent value, considering the pa- 
rameters of bed shape, can be represented by $[q]$, and the relationship between $[q]$ and the equivalent fuzzy variable $q$ is: $[q]=q(\mathrm{x} \mid h b l n)$. So the actual horizontal resistance $\mathrm{Q}$ of the ballast bed can be represented as follws:

$$
Q=[q] \cdot \xi
$$

where, $Q$ is the actual horizontal resistance; $[q]$ is the transversal resistance equivalent value; $\xi$ is the degree of looseness.

\subsection{Values of Non-Quantitative Bed Surface Parameters}

The values of the coefficient $\xi$ is related to the looseness of the ballast beds, and the values in different track bed conditions is different. The degree of looseness of the ballast bed is divided into three levels, dense, general and loose. The coefficients corresponding to each level are $\xi_{1}, \xi_{2}, \xi_{3}$, and the size meets the relationship of $1 \geq \xi_{1} \geq \xi_{2} \geq \xi_{3} \geq 0$. In addition, since the evaluation of appearance loosing degree subjectively be influenced by human factors, the value obtained in this case is not necessarily a fixed value, but it can also be a given range.

\subsection{Values of Quantitative Bed Surface Parameters}

The value of the bed thickness is affected by the operating conditions, the type of track, and the soil quality. The values are obtained through actual measurements. However, considering that bed's information is relatively stable when compared with other shape parameters, it can also be obtained from the retained data of the line design and construction. The value of the slope of the ballast bed is also relatively fixed. Generally, the line segment is taken as $1: 1.75$, and the station line is taken as 1:1.5.

Values of the height and width of the track bed is relatively broad and have different combinations. Appropriately increasing the shoulder width of the ballast can increase the weight of the ballast and then increase the lateral resistance. However, after the shoulder width has reached a certain level, the resistance value no longer increases. Therefore, there is a range of shoulder width values, 550 $\mathrm{mm} \geq \mathrm{b} \geq 300 \mathrm{~mm}$. In the same way, the height of the shoulder also has an impact on the improvement of the lateral resistance of the ballast bed. Because there is an internal friction angle limit in the turnout and the impact of ballast splashing on vehicle safety, there is also a limit value for the stack height, so the range of the stack height is: $l_{\text {lim }} \geq 1 \geq 0$.

\subsection{Other Cases in Which the Bed Surface Parameters Would Be Affected}

In addition to the conventional way of adjusting the bed surface parameters to improve the transverse resistance, the works department also uses the ground anchor rod to limit the lateral displacement of the track bed in special sections. In this case, the above method is inappropriate. In addition, some damaged rails and sleepers which removed from railway lines will be stacked next to the track bed. Therefore although the parameters of the bed surface have not been 
changed, it will undoubtedly have an impact on the lateral resistance. This case should be discussed separately.

In summary, the lateral resistance under the parameters of a specific track bed surface could be calculated in this way. Firstly it is necessary to determine the lateral resistance $\mathrm{q}$ according to the quantitative parameter conditions, and secondly determine the size of the correction coefficient according to the non-quantitative conditions. The product of the two is used as the final value of lateral resistance for the evaluation of the seamless line.

\section{Conclusions}

1) The lateral resistance of the ballast bed is the chief factor affecting the stability of the seamless track and its size would be affected by the parameters of the surface. The analogy of the trajectory of the lateral displacement of the trackless bed is proposed. It is proposed that the lateral resistance of the track bed is also ambiguous within the allowable lateral displacement limit, and equivalent randomization is performed.

2) For the lateral resistance of the ballast bed, this paper establishes a calculation framework containing a variety of parameters for the lateral resistance based on whether the parameters of the ballast surface quantitative or not. However, due to lack of actual measured data, the specific contents of the formula cannot be determined.

\section{References}

[1] Lu, Y.R. (2010) Research and Application of Seamless Track. China Railway Publishing House.

[2] Chen, X.F., Miao, P., et al. (1999) Stability Analysis of Continuous Welding Long Rail Track Stability. China Academic Journals Digest, No. 3.

[3] Zhang, X.M. and Chen, X.F. (2007) Stability Calculation and Parameter Sensitivity Analysis of the Seamless Loop Stability. Journal of the China Railway Society, No. 04, 70-73.

[4] Zhu, Z.W. and Lian, S.L. (2004) Sensitivity Analysis of Track Parameters Affecting the Stability of Continuous Welded Rails. China Railway Science, No. 01, 73-76.

[5] Li, Q.Y. and Chen, X.F. (2001) Stability Reliability of CWR Based on Fuzzy Lateral Migration Limit. China Railway Science, No. 03, 66-69.

[6] Chen, X.F., Yu, P., Xiang, Y.N. and Zhou, X.L. (1999) Extreme Probability Analysis of the Original Bending of Continuously Welded Rails. China Railway Science, No. 01 .

[7] Zhao, G.F. (1996) Engineering Structure Reliability Theory and Application. Dalian University of Technology Press, Dalian. 